\title{
Experiencia de Pittsburgh con la toxina botulínica A inyectable
}

\author{
F. de Miguel, M.B. Chancellor \\ Departamento de Urología. Universidad de Pittsburgh. Pittsburgh, PA, EEUU. \\ Actas Urol Esp 2006; 30 (3): 310-314
}

\section{RESUMEN}

EXPERIENCIA DE PITTSBURGH CON LA TOXINA BOTULÍNICA A INYECTABLE.

Presentamos la experiencia de un centro durante 6 años con el uso de la toxina botulínica A (TBON-A) en la vejiga, o en la uretra en 110 pacientes, con diversos trastornos del tracto urinario inferior.

Se inyectó a 110 pacientes (edad 19-82 años) TBON-A en la vejiga ( $=42)$ o la uretra $(\mathrm{n}=68), 35 \mathrm{~V}, 75 \mathrm{M}$. Entre los trastornos de la micción figuraban hiperactividad neurógena del detrusor y/o disinergia detrusor-esfínter, vejiga hiperactiva (VHA), hiperplasia benigna de próstata (HBP), obstrucción del cuello de la vejiga (OCV) y cistitis intersticial (CI). Hasta ahora, 27 pacientes han recibido varias inyecciones (hasta un máximo de 6) a intervalos de $\geq 6$ meses.

Todos los pacientes que recibieron inyecciones de TBON-A en la vejiga presentaban signos preoperatorios de contracciones involuntarias del detrusor en los estudios urodinámicos. El análisis de los 110 pacientes indica que en el $67,3 \%$ la incontinencia disminuyó o desapareció con el tratamiento. Los diarios indican que los síntomas diurnos y nocturnos de la micción disminuyeron. TBON-A fue eficaz a los 7 días y su eficacia se mantuvo durante al menos 6 meses. Las puntuaciones de sintomas en los análisis de CdV, las puntuaciones de síntomas específicos de la enfermedad también mejoraron. No ha habido complicaciones a largo plazo. En dos mujeres con esclerosis múltiple (EM) e incontinencia urinaria leve por estrés en el periodo basal, la pérdida de orina por estrés aumentó tras la inyección de TBON-A en el esfinter externo. En una mujer con EM que recibió una inyección en la vejiga, el volumen de orina residual aumentó de 78 a $155 \mathrm{ml}$. La paciente no tuvo que someterse a sondaje intermitente.

Ls inyección de TBON-A es una modalidad de tratamiento segura y prometedora para diversos trastornos del tracto urinario inferior tanto del músculo esquelético como del músculo liso. En la serie de pacientes estudiados, TBON-A es igual de eficaz en mujeres que en varones. Las inyecciones de TBON-A en la vejiga son eficaces no sólo para la hiperactividad neurógena del detrusor, sino también para la vejiga hiperactiva. El uso de TBON-A puede incluso considerarse para la CI.

Palabras clave: Toxina botulínica A. Tracto urinario inferior. Vejiga. Uretra. Próstata.

\section{ABSTRACT}

\section{PITTSBURGH EXPERIENCE WITH BOTULINUM TOXIN A INJECTION}

We report one institution's six-year experience using botulinum toxin A (BONT-A) in the bladder and urethra in 110 patients for a variety of lower urinary tract dysfunction.

110 patients (age 19-82) were injected with BONT-A into the bladder $(n=42)$ or urethra $(n=68), 35 \mathrm{M}, 75 \mathrm{~F}$. Voiding dysfunction included: neurogenic detrusor overactivity and/or detrusor sphincter dyssynergia, overactive bladder (OAB), benign prostatic hyperplasia (BPH), bladder neck obstruction (BNO) and interstitial cystitis (IC). Currently, 27 patients have undergone further injections (up to 6) at intervals $>6$ months.

All the patients with bladder BONT-A injection had preoperative evidence of involuntary detrusor contractions during urodynamic testing. Analysis of the 110 patients indicates that $67.3 \%$ reported a decrease or absence of incontinence. Diaries indicate a decrease in both day and night voiding symptoms. Efficacy occurred within 7 days and lasted for at least 6 months. Condition specific BOL symptom scores also demonstrated improvement. There have been no long-term complications. Two MS women with mild baseline stress urinary incontinence reported increased leakage with stress after BONT-A external sphincter injection. One MS woman who had a bladder injection had an increased residual urine from 78 to $155 \mathrm{ml}$. She did not have to perform intermittent catheterization.

BONT-A injection is a safe and promising treatment modality for a variety of lower urinary tract dysfunctions for both skeletal and smooth muscle dysfunction. In our series, BONT-A is equally effective in women as it is in men. Bladder injections with BONT-A are effective for not only neurogenic detrusor overactivity but also overactive bladder. BONT-A can even be considered for IC.

Keywords: Botulinum toxin A. Lower urinary tract. Bladder. Urethra. Prostate. 
$\mathrm{L}$ a toxina botulínica, aislada por primera vez por van Ermengem en 1897, es la toxina biológica más potente conocida para el ser humano. Actúa inhibiendo la liberación de acetilcolina en la unión colinérgica presináptica. En la clínica, los urólogos han utilizado las preparaciones comerciales de toxina botulínica tipo A (TBON-A) para tratar a pacientes con lesiones de la médula espinal que presentan disinergia vésico-enfinteriana (DVE, o disinergia detrusor-esfinter)(ASED) ${ }^{1}$. Más recientemente, se ha comunicado que el tratamiento de la vejiga hiperactiva de origen neurógeno y no neurógeno con TBON-A ha tenido éxito en varias partes del mundo. En este artículo resumimos nuestra experiencia en la Universidad de Pittsburgh con nuestros primeros 110 pacientes tratados con inyecciones de TBON-A en la uretra o en la vejiga ${ }^{2-4}$.

\section{MÉTODOS}

Los primeros 110 pacientes (35 varones y 75 mujeres, edad 19-82 años) tratados con inyecciones de TBON-A en nuestro centro recibieron las inyecciones en la vejiga $(n=42)$ o en la uretra $(n=68)$. Entre los trastornos de la micción figuraban esclerosis múltiple (EM), lesiones de la médula espinal (LME), ictus (ACV), vejiga hiperactiva (VHA) y cistitis intersticial (CI). Se hizo una historia médica completa y una exploración física a todos los pacientes y se les realizó un análisis urodinámico con vídeo antes de inyectarles TBON-A con arreglo a la directriz de la Sociedad Internacional de la Continencia utilizando un aparato Laborie Aquarius 120. La evaluación basal indicó que ningún paciente presentaba reflujo vesicouretral.

En condiciones de sedación leve o anestesia local con lidocaina, y tras recibir antibióticos en el preoperatorio, se inyectó a los pacientes TBON-
A (Botox ${ }^{\circledR}$, Allergan Inc., Irvine, CA, EEUU). Siguiendo estrictamente la recomendación del fabricante, la toxina se resuspendió en solución salina estéril sin conservantes inmediatamente antes del tratamiento. No se utilizó la electromiografía como guía para las inyecciones de TBON-A. Hasta ahora, 27 pacientes han recibido más de una inyección (hasta un máximo de 6) a intervalos de $\geq 6$ meses.

Técnica de inyección en la vejiga

Los pacientes reciben el tratamiento a nivel ambulatorio tras anestesia local con gelatina de lidocaína al $2 \%$ por vía intrauretral ó $30 \mathrm{ml}$ de lidocaína al $2 \%$ por vía intravesical durante 10 minutos. Utilizando una aguja para inyección flexible desechable de calibre 25 (Olympus Inc., Melville, NY, EEUU) que se inserta a través de un cistoscopio flexible, se inyectan 200 unidades de TBON-A diluidas en $20 \mathrm{ml}$ de solución salina sin conservantes por vía submucosa en 20 localizaciones del trígono y la base de la vejiga (Fig. 1). Todos los pacientes reciben antibióticos por vía oral en el preoperatorio y son sometidos a seguimiento de forma subjetiva por teléfono o mediante una entrevista en la consulta; además, se determina el volumen de orina residual después de la micción mediante ecografía de la vejiga durante las visitas clínicas.

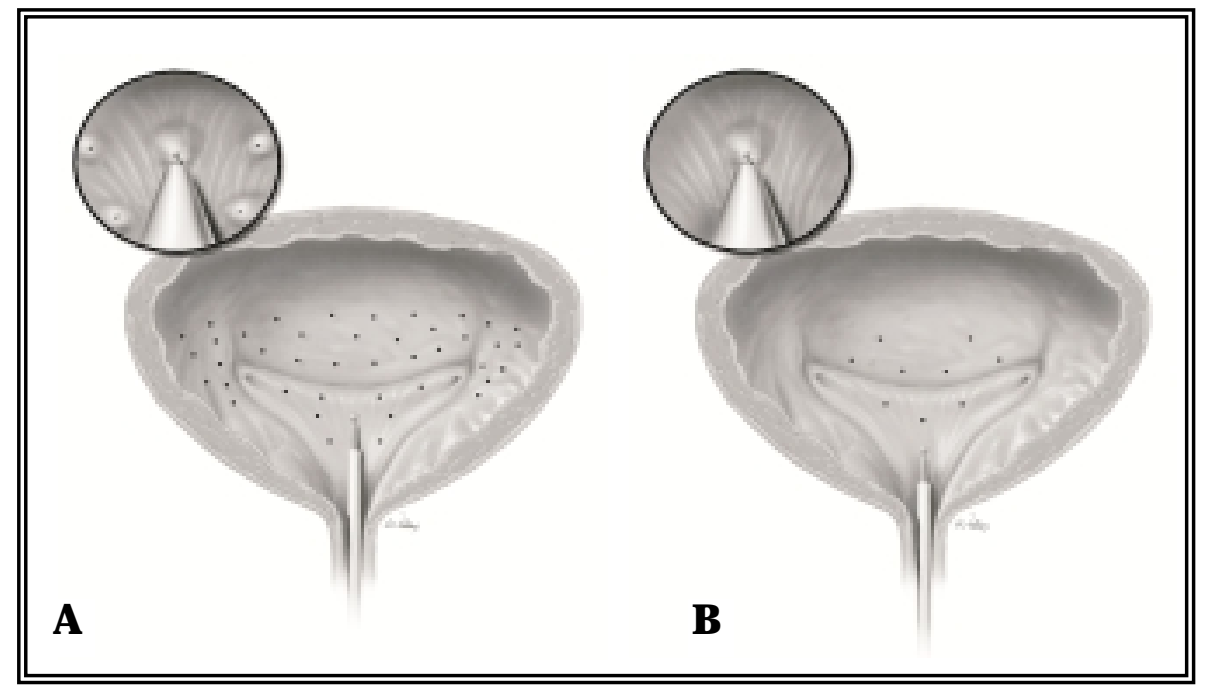

FIGURA 1. A) Se administraron aproximadamente 40 inyecciones de toxina botulínica $A(T B O N-A)$ en el trigono, la base y las paredes laterales de la vejiga para tratar principalmente a pacientes con vejiga hiperactiva de origen neurógeno. B) Se utilizó una técnica modificada de inyección de TBON-A en 10 puntos del trigono y la base de la vejiga para el tratamiento ambulatorio de pacientes con vejiga hiperactiva idiopática. 
Técnica de inyección en la uretra

Se utilizan un cistoscopio rígido y una aguja convencional de inyección de colágeno para cistoscopia para inyectar alícuotas aproximadamente iguales, $1 \mathrm{ml}$ cada una, de TBON-A en la parte profunda del esfínter externo en las posiciones de las 3, las 6, las 9 y las 12 en punto. Como alternativa, en algunas pacientes mujeres se utilizó una aguja espinal de calibre fino para inyectar TBON-A por vía periuretral. Los primeros tres pacientes recibieron un total de 80 unidades diluidas en $4 \mathrm{ml}$ de solución salina estéril. Debido a la ausencia de efectos secundarios en estos tres pacientes, la dosis de la toxina se aumentó a 100 unidades y 200 unidades diluidas en $4 \mathrm{ml}$ de solución salina estéril en todas las mujeres y varones restantes, respectivamente. Posteriormente se inyectaron 0,3 ml más de solución salina normal para administrar la toxina restante que quedaba en el espacio muerto de la aguja.

\section{RESULTADOS}

La eficacia máxima de la inyección de TBON-A se produjo entre siete y treinta días después del tratamiento y, en algunos pacientes, TBON-A alivió los síntomas durante más de 6 meses. No se observaron complicaciones sistémicas (como depresión respiratoria o hipostenia) ni locales (como incontinencia por estrés, retención urinaria o signos clínicos de reflujo vesicouretral [es decir, pielonefritis sintomática]) tras el tratamiento con TBON-A. El análisis de los pacientes indicó que en 74 de los 110 pacientes $(67,3 \%)$ la incontinencia disminuyó o desapareció (respuesta de 1 ó 2 en el cuestionario). Los datos de los 27 pacientes que recibieron al menos una segunda inyección de TBON-A mostraron que los síntomas mejoraron en todos ellos.

\section{Resultados de la inyección en la vejiga}

El seguimiento de 32 pacientes tras el tratamiento intravesical con TBON-A mostró una disminución del 40\% del número de micciones/24 horas ( $\mathrm{p}<0,05$ ), así como una reducción del 87\% en el número de pacientes que necesitaron utilizar pañales para la incontinencia (del 93\% al $12 \%$ ). Además, la capacidad cistométrica aumentó significativamente tras el tratamiento con TBON-A de 153 a $246 \mathrm{ml}(\mathrm{p}<0,05)$ en un segui- miento de 22 pacientes. No hubo un cambio significativo en el volumen de orina residual medio y ningún paciente presentó retención urinaria tras el tratamiento con TBON-A en la vejiga. En una mujer con EM que recibió una inyección en la vejiga, el volumen de orina residual aumentó de 78 a $155 \mathrm{ml}$, pero la mujer no tuvo que someterse a sondaje intermitente.

Nuestros resultados con las inyecciones de TBON-A en la vejiga son prometedores en una población de pacientes con síntomas de la micción recalcitrantes. Las únicas opciones para los pacientes en el momento del tratamiento eran neuromodulación del sacro o aumento de tamaño/desviación de la vejiga, y ambos procedimientos son invasivos. Es de destacar que todos los pacientes que respondieron bien a la inyección intramuscular inicial de TBON-A presentaron un resultado clínico similar tras recibir más inyecciones (hasta un máximo de seis inyecciones en la vejiga).

\section{Resultados de la inyección en la uretra}

Se realizó un seguimiento de seis meses a 2732 de 68 pacientes tras la inyección en el esfínter de la uretra. El volumen medio de orina residual después de la micción disminuyó de $250 \mathrm{ml}$ a 88 $\mathrm{ml}$ después del procedimiento $(\mathrm{p}<0,05)$. Además, la presión miccional máxima disminuyó de 81 a $52 \mathrm{~cm} \mathrm{H}_{2} \mathrm{O}(\mathrm{p}<0.01)$ y la capacidad cistométrica aumentó de 198 a $241 \mathrm{ml}$. La incidencia de retención urinaria que requirió sondaje se redujo en un $80 \%$ y las tasas de infección disminuyeron en paralelo con la mejoría de la función neurológica (es decir, menos espasticidad y fatiga). En dos mujeres con EM e incontinencia urinaria leve por estrés en el periodo basal, la pérdida de orina por estrés aumentó tras la inyección de TBON-A en el esfínter externo, pero ambas pacientes también presentaron mejoras significativas de la disinergia vésico-esfinteriana, una reducción del volumen de orina residual, una mejora del flujo urinario y una reducción de la incontinencia de urgencia y de la frecuencia de micciones diurnas y nocturnas.

Justo antes de administrar las inyecciones de TBON-A en el esfinter de la uretra, se mezclan 100-200 unidades de toxina botulínica con aproximadamente $4 \mathrm{ml}$ de solución salina. Es impor- 
tante no agitar el vial, ya que esto puede romper el enlace disulfuro existente entre las cadenas ligera y pesada y provocar que la toxina sea ineficaz. Utilizando una aguja de inyección de colágeno (preferimos la Cook Urologic ${ }^{\circledR}$ [Bloomington, IN, EEUU] debido a que la punta es más afilada) a través de un cistoscopio convencional o una aguja espinal de calibre fino para la aplicación periuretral, se administran inyecciones de $1 \mathrm{ml}$ cada una en las posiciones de las 3, las 6, las 9 y las 12 en punto en el esfínter estriado. Las inyecciones deben administrase a una mayor profundidad que las inyecciones de colágeno para alcanzar las terminaciones nerviosas que inervan el músculo esquelético. También se enjuaga la aguja con $0,3 \mathrm{ml}$ de solución salina al final del procedimiento para asegurarse de que no queda nada de toxina en el espacio muerto de la aguja.

\section{TBON-A para la HBP}

Recientemente, se ha inyectado TBON-A en la próstata como tratamiento de la HBP. Hemos publicado los resultados de una serie de 16 varones con HBP sintomática y un índice de flujo urinario máximo $<12 \mathrm{ml} / \mathrm{s}$ que recibieron inyecciones de Botox (100 U/4 ml) en la próstata ${ }^{5}$. Se administró una inyección del mismo volumen (2 ml) en cada lóbulo de la próstata. No se observaron efectos secundarios locales o sistémicos en ningún paciente. Todos los pacientes presentaron una mejoría subjetiva aproximadamente al cabo de una semana después de las inyecciones, alcanzaron el efecto máximo al cabo de un mes y la mejoría se mantuvo durante un periodo de seguimiento medio de 10 meses. El volumen prostático medio, la puntuación de síntomas y el índice de calidad de vida disminuyeron significativamente en un $13,3 \%$ (de 19,6 $\pm 1,2 \mathrm{ml}$ a $17,0 \pm 1,1$ $\mathrm{ml}$ ), un $52,6 \%$ (de $18,8 \pm 1,6$ a $8,9 \pm 1,9$ ) y un $44,7 \%$ (de $3,8 \pm 0,3$ a $2,1 \pm 0,3$ ), respectivamente. El indice de flujo urinario máximo aumentó significativamente en un $39,8 \%$ (de 7,3 $\pm 0,7 \mathrm{ml} / \mathrm{seg}$ a $11,8 \pm$ $0,8 \mathrm{ml} / \mathrm{seg}$ ). En 2 pacientes a los que se les hizo una biopsia 1 mes después de la inyección de TBON-A, la tinción con TUNEL mostró un aumento de la apoptosis no sólo en el componente glandular, sino también en el componente estromal del tejido prostático. Estos datos clínicos indican que TBON-A alteró el efecto trófico del sistema nervioso autónomo sobre la glándula prostática humana e indujo la apoptosis celular. Sin embargo, en 4 de los 16 pacientes el índice de flujo urinario máximo aumentó y los síntomas mejoraron sin que se redujera el volumen de la próstata.

La inyección de TBON-A en la próstata es un tratamiento prometedor para los varones con HBP sintomática. Los mecanismos responsables del alivio de los STUI mediante la inyección de TBON-A en la próstata puede que no dependan totalmente de la reducción del volumen de la próstata. La inhibición del tono muscular liso y de la función sensorial aberrante podría desempeñar un papel importante. Estos resultados constituyen un avance verdaderamente importante en la investigación de la toxina botulínica en el campo de la urología.

\section{CONCLUSIONES}

Se ha demostrado que la toxina botulínica es un tratamiento seguro y eficaz para diversos trastornos motores somáticos y del sistema nervioso autónomo. Actualmente, los urólogos estamos consiguiendo éxitos clínicos con las inyecciones intramusculares de TBON-A en la uretra y la vejiga en el tratamiento de la DVE, trastornos de la espasticidad del suelo de la pelvis y la vejiga hiperactiva $^{4,5}$ (Figura 2). En 2005 están a punto de

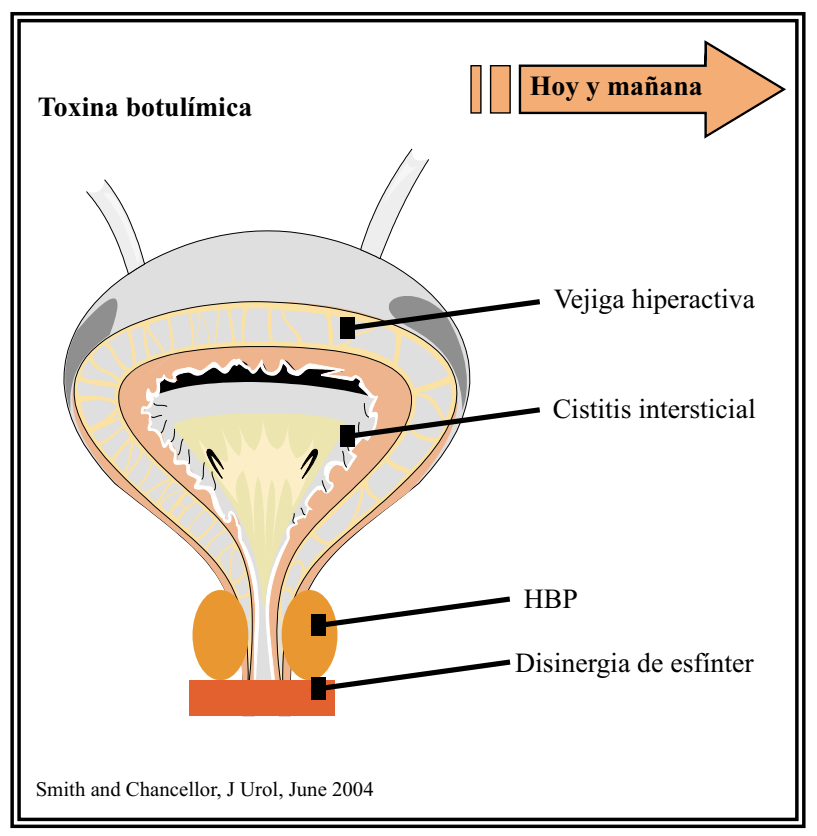

FIGURA 2. Aplicaciones actuales y futuras de la inyección de toxina botulinica en el tracto urinario inferior. 
iniciarse ensayos clínicos en fases 2 y 3 aprobados por la FDA. No se puede negar que el ser humano tiene ingenio, debido a que ha transformado la toxina letal de Clostridium botulinum en un medicamento terapéutico moderno.

\section{REFERENCIAS}

1. Dykstra DD, Sidi AA, Scott AB, Pagel JM, Goldish GD. Effects of botulinum A toxin on detrusor-sphincter dyssynergia in spinal cord injury patients. $J$ Urol. May 1988;139(5):919-922.

2. Smith CP, Nishiguchi J, O'Leary M, Yoshimura N, Chancellor MB. Single-institution experience in 110 patients with botulinum toxin A injection into bladder or urethra. Urology. Jan 2005;65(1):37-41.

3. Phelan MW, Franks M, Somogyi GT, Yokoyama T, Fraser MO, Lavelle JP, et al.. Botulinum toxin urethral sphincter injection to restore bladder emptying in men and women with voiding dysfunction. J Urol. Apr 2001;165(4):11071110.

4. Smith CP, Chancellor MB. Emerging role of botulinum toxin in the management of voiding dysfunction. $J$ Urol. Jun 2004;171(6 Pt 1):2128-2137.
5. Chuang YC, Chiang PH, Huang CC, Yoshimura N, Chancellor M.B: Botulinum toxin type A improves benign prostatic hyperplasia symptoms in patients with small prostates. Urology 2005;66:775-779.

6. Schurch B, Hauri D, Rodic B, Curt A, Meyer M, Rossier AB. Botulinum-A toxin as a treatment of detrusor-sphincter dyssynergia: a prospective study in 24 spinal cord injury patients. J Urol. Mar 1996;155(3):1023-1029.

7. Schurch B, de Seze M, Denys P, Chartier-Kastler E, Haab F, Everaert K, Plante P, et al. Botox Detrusor Hyperreflexia Study Team. Botulinum Toxin Type a Is a Safe and Effective Treatment for Neurogenic Urinary Incontinence: Results of a Single Treatment, Randomized, Placebo Controlled 6-Month Study. J Urol. Jul 2005;174(1):196-200.

Dr. F. de Miguel

Department of Urology

3471 Fifth Avenue. Suite 700

Pittsburgh, PA 15213 - EE.UU.

E-mail: demifx@upmc.edu

(Trabajo recibido el 1 de diciembre 2005) 